\title{
El sistema político mexicano en el cambio democrático en América Latina
}

\author{
Manuel Alcántara Sáez \\ UNIVERSIDAD COMPLUTENSE
}

"En estos tiempos de transformaciones no es posible gobernar un país como el nuestro con hipótesis o prácticas políticas de decenios anteriores."

\section{INTRODUCCIÓN}

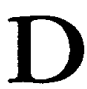

esde la segunda mitad de la década de 1970, la vida política latinoamericana ha contemplado el fenómeno del tímido, pero paulatino, asentamiento de regímenes políticos democráticos que se han generalizado a prácticamente todos los países de la región diez años más

1 Palabras pronunciadas por Carlos Salinas de Gortari ante el Congreso de la Unión el 1 de noviembre de 1991, Conercio Exterior, vol. VL, núm. noviembre 1991, p. 1074. tarde. Si bien situaciones parecidas han acaecido en distintos momentos de la historia latinoamericana, como sucedió en la década inmediatamente anterior a la gran crisis de 1929 y al periodo de los tres lustros que siguieron a la segunda guerra mundial, el actual es un momento cuantitativa y cualitativamente diferente.

Por primera vez coinciden en el tiempo un número de países superior a la veintena que son gobernados por líderes elegidos por sufragio popular; procesos que han pasado por todo tipo.de filtros y condiciones para asegurar su bondad y que han sido traducción de la libre expresión de la voluntad de los ciudadanos.

En México, cuya difícil equiparación con las restantes situaciones naciona- 
les es una constante desde la fundación de su actual régimen político, los procesos democratizadores vienen asimismo afectando a este último desde 1977, aunque con un ritmo mucho más cadencial. En la actualidad aparece sumergido en un marco donde las profundas transformaciones llevadas a cabo en el entramado económico han dejado atrás a las más apocadas realizadas en el político.

A la vez, los fenómenos que han tenido lugar en los países centro y esteeuropeos han conformado una situación en la que, completamente desaparecido el denominado socialismo real, el avance de formas democráticas es incuestionablemente abrumador. En esta peculiar situación, América Latina, y México en particular, se encuentran insertos en un fenómeno de amplitud universal que sobrepasa la mera extensión regional. No obstante, la aparición de la pléyade de reformismo económico para la mayoría de los casos y de la senda democratizadora, que en menor medida concierne a México, ha sido significativamente anterior a la caída de los sistemas comunistas europeos, estando también relacionada con el agotamiento del modelo de Estado seguido.

\section{DE LAS TIPOLOGÍAS EN POLÍTICA COMPARADA DE LA DÉCADA DE} 1960 Y IA AUSENCIA LATINOAMERICANA

Desde un punto de vista analítico, siguiendo patrones de estudio de política comparada, ${ }^{2}$ aparece una conse-

2 Un primer esbozo aproximativo de lo cuencia inmediata del nuevo estado de cosas, cuyo ámbito teórico es de gran relevancia y que acompaña a este proceso planetario: se trata de la influencia que este nuevo estado necesariamente va a tener que producir en el estudio de las formas de gobierno y en las tipologías que de las mismas se han derivado. Sin embargo, es fácilmente constatable que la ausencia de los casos latinoamericanos fue la nota dominante en el seno de las taxonomías elaboradas por la ciencia política en torno a la década de los años sesenta.

A este respecto, un primer elemento que se presentaba de manera palmaria ante cualquier observador, era la estrechez de las categorías de las formas de gobierno democráticas establecidas después de 1945 y mantenidas sin interrupción durante ya casi medio siglo. Los estudios de política comparada se centraron en un número muy reducido de casos nacionales que cumplían los requisitos de ser considerados como poliarquías. En este sentido, los análisis que afloraron en la citada década fueron pioneros en la aproximación desde dicha disciplina al estudio del gobierno y a las interrelaciones existentes entre éste y las distintas fuerzas políticas, sociales y económicas. Estos trabajos ya recogían experiencias políticas de más de dos décadas de los sistemas asentados después de la guerra pero, fundamentalmente, basaban sus presupuestos teóricos en los esquemas existentes en dos de los países victoriosos de la contienda munciial (Estados Unidos

aquí desarrollado se encuentra en Alcántara Sáez, “¿Dónde?", 1991, pp. 9-13. 


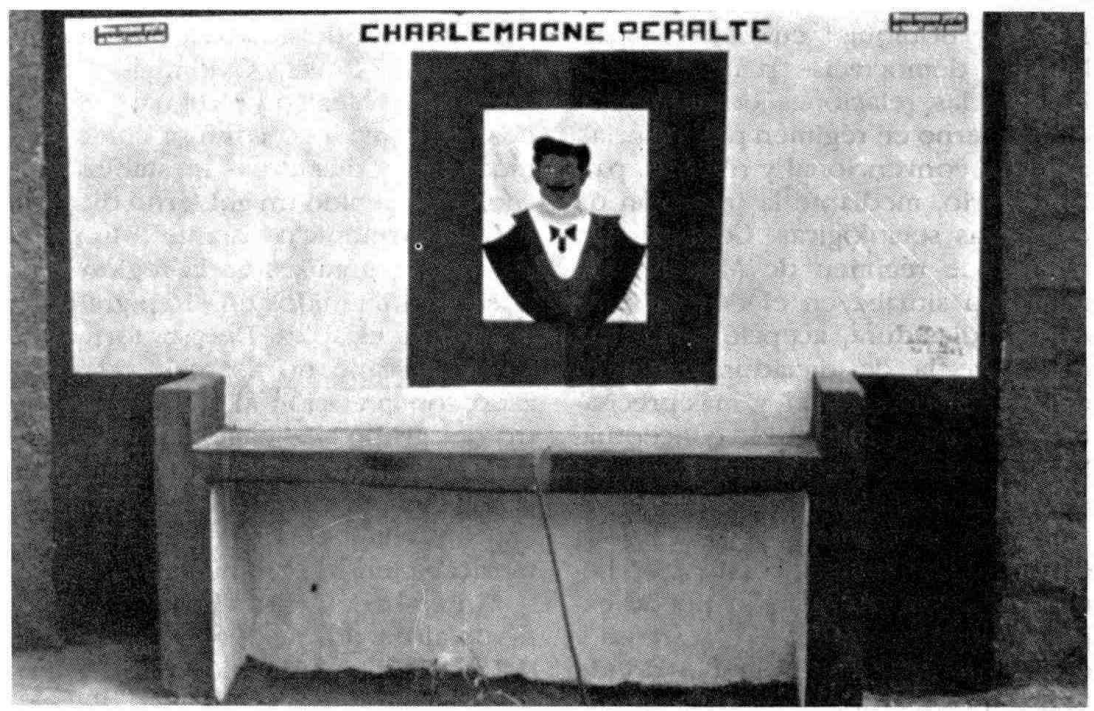

y Reino Unido), cuya forma diferente de organizar la política fue elevada a la categoría de modelo (presidencialismo y parlamentarismo). De esta manera, la reflexión empírica se centraba en dos casos sobresalientes y en un número que apenas llegaba a la quincena de fórmulas menores, cuya comprensión, de cualquier manera, se iluminaba con las categorías aprendidas de los primeros, estando su visión lógicamente impregnada de un fuerte contenido eurocéntrico. En la otra acera se daba cabida a una prolija clasificación de los regímenes no democráticos, en la que se insertaban la gran mayoría de los países de América Latina mediante su encasillamiento en diferentes subcategorías.

Maurice Duberger estableció su co- nocida clasificación en la que, dentro de las denominadas democracias liberales, distinguía entre los regímenes de tipo europeo y el régimen de Estados Unidos; ${ }^{3}$ al introducir en su marco las variables de división de poderes y de sistema de partidos políticos existente, señalaba una notoria diferencia entre lo que denominaba los regímenes británicos y los de tipo continental, en los que hacía de nuevo una distinción por su parlamentarismo mayoritario, por su parlamentarismo no mayoritario y por el semipresidencialismo. De cualquier manera, lo que Duverger había realizado era avanzar en el análisis jurídico-institucional de

3 Duverger, Instituciones, 1970, pp. 242324. 
Prélot y Boulouis ${ }^{4}$ cuando clasifica. ban las democracias por la naturaleza de las relaciones del pueblo y del gobierno en régimen presidencial, régimen convencional y régimen parlamentario, mediante la inclusión de categorías sociológicas. Con relación al tipo de régimen de México, Du. verger lo situaba en el denominado de semidictadura, acepción intermedia entre la de dictadura y la de democracia occidental, y, más precisamente, en una subdivisión conceptualizada como "régimen de partido muy dominante"s (para diferenciarlo de los "regímenes bajo tutela militar"). Esta denominación, que se basaba en el hecho de que "la oposición puede expresarse, casi siempre, en la prensa y en la tribuna parlamentaria" y donde "existen elementos democráticos reales, aunque esencialmente se trate de una dictadura de partido", se complementaba con el "carácter semiconservador" del régimen, habida cuenta de sus "reformas socializantes", la importancia del sector público, "la voluntad de independencia nacional" y el mantenimiento de una base económica esencialmente capitalista.

Al tener en cuenta el grado de desarrollo económico, así como una probada continuidad democrática en su forma de gobierno desde la primera guerra mundial, Seymur M. Lipset estimaba que solamente trece países podían ser considerados como democracias estables. Entre ellos ninguno estaba localizado en América Latina, 51-118.

4 Prélot y Boulouis, Institutions, 1987, pp

5 Duverger, Instituciones, p. 526. región en la que situaba a siete países (Argentina, Brasil, Colombia, Costa Rica, Chile, México y Uruguay) definidos de forma algo confusa como democracias y dictaduras inestables por "no haber tenido un gobierno dictatorial regularmente constante", ${ }^{6}$ frente a los restantes países de la región que quedaban incluidos en el epígrafe de dictaduras estables. De esta forma, el caso mexicano no aparecía categorizado con precisión al situársele dentro del grupo de "naciones con régimen inestable", algo que se alejaba de la realidad por cuanto que, si un factor era consustancial con la praxis política mexicana, era el continuismo estable.

Sin embargo, para el escenario de. finido alrededor de 1960 y alejándose del requisito de un periodo de estudio de tan prolongada estabilidad, Robert Dahl, al integrar las variables de' liberalización (debate público) y de representación (participación), establecía el número de poliarquías representativas en 24 de las cuales, en el entorno latinoamericano, solamente reconocía a tres: Costa Rica, Chile y Uruguay (de las que solamente se mantendría la primera tras la vorágine del autoritarismo militar).$^{7}$ México quedaba fuera de este nuevo marco.

Por su parte, Samuel Huntington esbozaba una tipología de sistemas políticos ${ }^{8}$ que, al recoger un entrecru. zamiento de la ratio existente de institucionalización con la variable de par-

6 Lipset, Hombre, 1977, pp. 28 y 29.

7 Dahl, Poliarquia, 1989. En las páginas 85 y 86 aparece la enunciación de los países democráticos de conformidad con las reglas de la poliarquía.

${ }^{8}$ Huntington, Political, 1968, pp. 78-83 
ticipación política, generaba un cuadro más sofisticado que los anteriores, en el que los países latinoamericanos se disolvian en el interior de las nueve categorías establecidas.

En el caso mexicano, la institucionalización se llevaba a cabo por intermedio del partido político oficial que asumía las funciones de los militares y del papel sustancial de los sindicatos existentes en otros países de la región. En estas tres aproximaciones teóricas, la vertebración de las formas políticas latinoamericanas, el ámbito más genérico de las democracias políticas, no incidió en tipología alguna de estas últimas, quedando inmersas en el cuadro genérico y teórico del presidencialismo.

\section{LOS INTENTOS DE TIPOLOGIZACIÓN DE LA} REALIDAD POLÍTICA LATINOAMERICANA

Jacques Lambert ${ }^{9}$ desarrolló desde un más estricto conocimiento de la realidad latinoamericana una forma peculiar derivada de la enunciada en el último párrafo del epígrafe anterior, a la que denominó la "preponderancia presidencialista", la cual, en su opinión venía a enmascarar auténticas dictaduras constitucionales. Según su visión, el sistema político mexicano se situaba entre los de aquellos países desigualmente desarrollados y de estructura social dual, con un nivel intermedio de evolución y con relativo equilibrio entre los segmentos evolucionados y los arcaicos de sus sociedades; sin embargo, su compo-

\footnotetext{
9 Lambert, América, 1973, pp. 518-553.
}

sición étnica, donde la población india había desarrollado siempre cierto dinamismo, y la influencia perturbadora de Estados Unidos que le "obligaba al cambio para sobrevivir", ${ }^{10}$ aportaban a México un grado de diferenciación notable con respecto a otros casos latinoamericanos equiparables (Brasil, Colombia y Venezuela). Todo ello condicionaba al presidencialismo, extremadamente reforzado por el aparato del partido, y que únicamente se veía limitado por el estricto cumplimientrs de la cláusula constitucional de la no reelección.

En torno a esta fuerte impronta presidencialista, Federico Gil ${ }^{11}$ señulaba la existencia de "importantes desviaciones del principio de separación", lo que le llevaba a afirmar que en América Latina la separación de poderes "no ha sido observada nunca estrictamente", traduciéndose en un acentuamiento del "predominio del poder ejecutivo". Circunstancia que en el caso de México, volvía a insistir este autor, se complementaba con el carácter "prácticamente unipartidista"12 del país.

Un intento diferente por conformar una tipología de los sistemas políticos de América Latina, con evidentes problemas de interpretación desviada y claramente tendiente a la confusión, llevó a Eldon Lanning a establecer una clasificación en la que se entrecruzaban dos características básicas. ${ }^{13}$ La primera hacía referencia al rasgo dominante de las bases orga-

10 Ibid., p. 102.

11 Gil, Instituciones, 1966, p. 74.

.12 Ibid., p. 134.

13 Lanning, "Typology", 1974, p. 379. 
nizativas de los sistemas, en las que se recogían unos de ámbito horizontal de intereses funcionales donde participaban grupos de interés y otros de orden jerárquico que definían relaciones personales. Complementariamente, la segunda característica concernía a las relaciones de poder entre las autoridades de los gobiernos centrales y los grupos políticamente más relevantes, la cual daba paso a tres situaciones: de dominio de grupo, de equilibrio y de dominio de la autoridad. Según todo ello, se generaban seis tipos básicos de sistemas políticos en los que México quedaba incluido junto con Chile, Costa Rica y Venezuela en el casillero del concepto denominado de "demanda de intereses" definido por el cruce de las variables de ámbito funcional y de equilibrio de poder: En relación con los análisis ofrecidos anteriormente, sorprende que este autor incluyese a México en este nicho que identificaba al "sistema moderno de. mocrático o poliárquico". ${ }^{14}$

Frente a este análisis, una profundización más compleja incorporaba la necesidad de afrontar un estudio global en el que se tuvieran en cuenta cierto número de dimensiones de variación. Martin C. Needler ${ }^{15}$ señalaba la necesidad de contar, al menos, con cinco dimensiones. La primera se refería al trasfondo socioeconómico, en el que se debían contabilizar aspectos como la geografía, los factores económicos, la composición étnica y sus mutuas interacciones. La segunda recogía factores históricos, con espe-

14 Ibid., p. 381.

is Needler, Political, 1964, pp. 513-522. cial énfasis en las acciones innovadoras de los estadistas, las no premeditadas consecuencias de las guerras y el impacto de comportamientos seculares de la economia mundial. La tercera concernía al papel desempeñado por los grupos de interés y los partidos en los procesos políticos. Por fin, las dos últimas dimensiones se centraban en la estructura gubernamental y en los temas dominantes de políticas públicas. Bajo esta variada perspec. tiva, "México se veía definido por diferentes elementos que articulaban un modelo complejo cuya evaluación final era favorable, según este autor, en comparación con los restantes países vecinos". ${ }^{16}$

LAS APORTACIONES MÁS RECIENTES AI. ESTUDIO DE LOS SISTEMAS POLÍ́TICOS comparados

La afirmación de Alain Touraine ${ }^{17}$ de que los sistemas políticos latinoamericanos "no son formas imperfectas del modelo clásico europeo o norteamericano, combinando éste con los restos de una organización social y política llamada tradicional" abre la posibilidad de incorporar a su estudio las formas de movilización y de acción colectiva. Según todo ello, aparecen en lugar central tres principios de análisis: "la subordinación de la acción social a la intervención del Estado; la combinación, en toda acción política, de la defensa de los intereses económicos, de la lucha contra la dominación ex-

16 Ibid., 32.

17 Touraine, América, 1989, p. 157. 
terior y de la voluntad de integración nacional, y de la desarticulación de la acción económica, de la organización política y de las expresiones ideológicas". En definitiva, se trata de adecuar los tipos de sociedad con las formas de modernización. Todo ello hace que entrañe "cierta indiferenciación entre el Estado y el sistema político", ${ }^{18}$ clásica en la vida latinoamericana y que en México cobra especial relevancia por el carácter globalizante de aquél y la usurpación y utilización de elementos por los que se regula o manipula a los actores sociales (partido político y sindicatos). En este país, por otra parte, el enfrentamiento contra la dependencia externa y la búsqueda de una firme integración nacional, por mediación ambas esferas de la actuación del Estado, ha sido la nota dominante durante más de medio siglo.

Asumiendo la posibilidad de integrar los sistemas de autoridad con los tipos de actores estatales dominantes, Gonzalo Varela ${ }^{19}$ plantea que la diferenciación de aquéllos en tres situaciones básicas: la de Estado (cuando existe una autoridad política centralizada, autónoma y estable), la de arbitraje (en el momento en que uno o más actores son capaces de imponer un equilibrio inestable al sistema político) y la de fragmentación (cuando no existe un centro institucional autónomo ni hay acuerdo entre elites), debe combinarse con unos actores estatales constituidos específicamente en función de la gestión pública (y a los que se van añadiendo de ma-

18 Ibid., p. 137.

19 Varela, "Análisis", 1990, pp. 144-145. nera gradual, los partidos políticos, el sector corporativo, la burocracia pública civil y las fuerzas armadas). En esta conceptualización, México "sería el caso de un Estado apoyado en un pacto corporativo". ${ }^{20}$

A partir de una evaluación con cier. tos elementos comunes, Thomas E. Skidmore y Pete $\mathrm{H}$. Smith ${ }^{21}$ señalan que un esquema analítico adecuado para el estudio de la política latinoamericana debería recoger una guarnición de actores sociales en la que se diera cabida en primer lugar a la estructura y alineación de las clases sociales, según dos dimensiones separadas: la de la posición urbano-rural y la del estatus (alto, medio y bajo). En segundo término debería tenerse en cuenta al actor social adicional en que consiste el sector exterior y en el que se incluye tanto a las inversiones y a las corporaciones privadas como a los gobiernos extranjeros y a las maquinarias militares. Por último, los actores sociales compiten por el control de las instituciones más importantes, entre las que el Estado aparece como más relevante, sin dejar detrás a la Iglesia católica. Según esta percepción, los gobiernos posrevolucionarios mexicanos obtuvieron apoyo popular de los obreros y de los campesinos; y, bajo Cárdenas, pusieron en marcha una estrategia para tratar con las masas por la que el Estado organizaría a estas clases trabajadores para mantenerlas aparte. El PRI desarrolló sectores separados para obreros y campesinos, "re-

$20 \mathrm{Ibid}$, p. 146.

${ }^{21}$ Skidmore y Smith, Modern, 1989, pp. 372 375. 
flejando la obsesión del régimen de poner proa a cualquier política clasista espontánea". ${ }^{22}$

Otra manera de enfrentarse al análisis viene derivada del estudio de las tendencias existentes en los sistemas políticos que llegan a favorecer ciertos intereses de clase, regionales o politicos, a expensas de otros. De acuerdo con la tesis de Howard J. Wiarda y Harvey $\mathbf{F}$. Kline, ${ }^{23}$ los sistemas políticos latinoamericanos recogen cuatro tendencias que se han ido destilando a lo largo de su historia: en primer lugar se basan en la idea de asumir una amplia comunidad de intéreses en el seno de la nación y de compartir valores y perspectivas populares que fueron probablemente más fuertes en el siglo anterior que en el presente. En segundo término, existe una fuerte tendencia en favor del statu quo político, lo que no quiere decir, según estos autores, que el cambio no pueda ocurrir nunca, sino que más bien tiende a darse en el marco de límites cuidadosamente definidos y de acuerdo con las reglas del juego. La tercera tendencia se refiere al sistema de clases existente, por el que se admite la inclusión de nuevos grupos pero únicamente con la condición de que acepten las reglas establecidas por los tradicionales detentadores del poder (la elite y los sectores medios ascendentes). Finalmente, hay tanto la necesidad como la aceptación de un crecimiento económico sostenido para asegurar cierta capacidad en el reparto de la riqueza, aunque para

22 Ibid., p. 381.

23 Wiarda y Kline, Latin, 1990, pp. 116-118. ello el acomodativo y cooptativo proceso político debe funcionar.

Existe, por el contrario, la posibilidad de realizar una aproximación menos general centrándose exclusivamente en el papel desempeñado por los partidos en el seno de los sistemas políticos, aun partiendo del reconocimiento de la limitada y desigual influencia de estas instituciones en la vida política nacional. En este sentido, Ronal $H$. McDonald y J. Mark Ruhl ${ }^{24}$ establecen una clasificación en la que dan cabida a cuatro categorías diferentes: aquélla en la que los partidos políticos son "actores dominantes"; otra en la que son "actores primarios pero no dominantes"; una tercera en la que son "actores secundarios", $y$ una última en la que son "actores marginales". México queda encuadrado en la primera de las categorías, situándose en segundo lugar solamente detrás de Cuba, una vez aplicado el índice de fraccionalización de Rae, ${ }^{25}$ circunstancia por la que los citados autores llevan a definir a su sistema de partidos como hegemónico.

Desde una perspectiva muy diferente, y dejando al margen los estudios que exclusivamente se centran en la región, Arend Lijphart, ${ }^{26}$ al analizar 22 tipos diferentes de regíme. nes democráticos ininterrumpidos entre 1945 y 1980 , cruzando las variables formas de gobierno y cohesión social (homogeneidad-heterogeneidad), no recoge a ningún país latinoamericano. De esta forma, la tipología

\footnotetext{
24 McDonald y Ruhl, Party, 1989, pp. 1-3.

25 Ibid., p. 10.

${ }^{26}$ Lijphart, Democracias, 1987.
} 
que establece sobre democracias mayoritarias y democracias de consenso, resultado del citado cruce, se escapa al influjo de la realidad política latinoamericana. Por otra parte, la presencia de peculiaridades en la forma presidencialista de gobierno en la región en periodos de dilatada vigencia democrática, llega incluso a poner en duda la validez de la teoría de este politólogo. En aquellos casos naciona. les más significativos como serían los de Costa Rica, Venezuela, Uruguay y, con las limitaciones pertinentes, Colombia, Honduras y la República Dominicana, se entrecruzan algunas de las características que definen los dos modelos de Lijphart y que pertenecen a universos excluyentes. Así, en estos países latinoamericanos, aparecen algunos de los elementos propios del modelo de democracia mayoritaria, como son la concentración del poder ejecutivo, la fusión de poderes y dominio del gabinete, el bipartidismo, el gobierno centralizado y la democracia exclusivamente representativa. Pero también recogen aspectos del modelo de democracia de consenso como son: el bicameralismo equilibrado, la representación propor. cional y la constitución escrita y el veto de la minoría. Ahora bien, esto no es óbice para que Jonathan Hartlyn haya podido analizar con éxito el carácter consociacional del sistema colombiano del Frente Nacional. ${ }^{27}$

El escenario existente en 1992 pone suficientemente de relieve lo inadecuado del estudio de la nueva situación siguiendo los parámetros ela-

27 Hartlyn, Politics, 1988. borados sobre una muestra tan reducida de casos nacionales como la realizada por Lijphart y, con anterioridad, por los otros autores citados. Además, la evolución interna de muchos de los países occidentales no latinoamericanos que han servido de guía hasta la fecha, han visto también cómo los viejos paradigmas explicativos de su tramoya política peculiar han sido sustituidos por otros nuevos. De esta manera, se difuminan, entre otras, las variables religiosa y de clase social, mientras que toman vigor la lingüística, la ecología y aquellas otras que enfatizan el papel que pudieran desempeñar las "oportunidades críticas" en su capacidad de modelar el futuro de la política. ${ }^{28}$ Además, en el terreno institucional la confusión se acrecienta entre las formas puras de parlamentarismo y de presidencialismo, así como en la im. posibilidad de conformar mayorías estables en sociedades plurales.

\section{¿VINO NUEVO EN ODRES VIEJOS?}

En América Latina, donde los procesos de transición política han dejado su secuela que afecta directamente a sus nuevos sistemas políticos, se constata el necesario replanteamiento de muchos de los intrumentos utilizados para el estudio de la política desde una aproximación comparada. Se observa que la actual situación de éxito de la mayoría de los procesos demo-

28 Collier y Collier, Shaping, 1991, pp. 30 y ss. Se refieren a "critical junctures" (en el original) a la hora de establecer un sugestivo modelo de análisis del desarrollo polírico en ocho países latinoamericanos. 
cratizadores iniciados a finales de la década de los años setenta comporta un escenario específico en el que se dan cita cuatro situaciones nuevas y una quinta que es reiterativa en la tradición política del área. A pesar de todo ello, es notable comprobar que el caso mexicano cobra vida autónoma presentándose bajo claves de gran especificidad y diferenciación que contrastan con las de los restantes países.

En primer lugar surge una idea, que paulatinamente se va generalizando, por la que las categorías tradicionalmente utilizadas para encontrar taxonomías de los sistemas políticos en torno a los ejes parlamentarismopresidencialismo, democracia de consenso-democracia mayoritaria y sistema de representación proporcional sistema de representación mayoritaria, entre otras, quedan obsoletas frente a las nuevas realidades políticas conformadas. En consecuencia, se deben repensar unas nuevas, de conformidad con los casos reales que se van sucediendo y que cuentan, ya en gran número, con al menos dos periodos presidenciales de nuevos gobiernos democráticos. El encasillamiento actual de los regímenes latinoamericanos como unos de carácter presidencialista es equívoco por cuanto que la casuística constitucional es muy variada, llegando a generar cuadros en los que el presidente puede disolver una cámara (Perú y Uruguay), en los que el legislativo tiene potestad de censurar a ministros (Perú, Ecuador), o en los que el legislativo es facul-

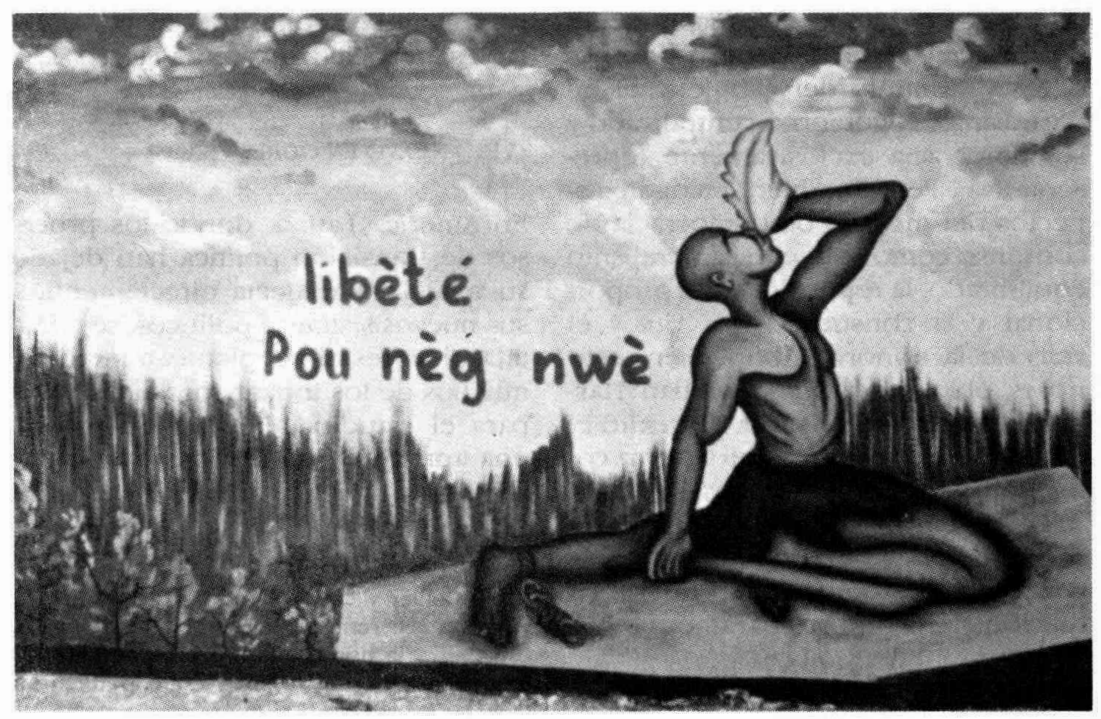


tado para elegir al presidente cuando éste no alcanza la mayoría absoluta en los comicios (Bolivia). Igualmente, las variables definidas para contrapolar el carácter mayoritario frente al de consenso del sistema no son rigurosamente aplicables. En cuanto a la bipolaridad en el sistema de representación, es artificial ya que la ma. yor parte de los casos nacionales han adoptado el sufragio proporcional con diferentes tipos de circunscripciones que en algún país llegan incluso a mezclarse (en Ecuador se combinan la circunscripción nacional con las regionales). No obstante, México se aísla de esta situación regional manteniéndose aferrado a un sistema presidencialista incuestionable, en el que, además, las reformas electorales de 1991 desvirtúan los efectos de una forma mixta (de sufragio mayoritario y proporcional) de elección para el Congreso de los Diputados, toda vez que se otorga directa y automáticamente la mayoría del mismo al partido que apenas supere un tercio del voto popular.

El papel de los partidos políticos en la generalidad de los países de América Latina ha visto modificado sustantivamente su significado, de manera que aparece como algo nuevo en sus características a la hora de integrar y de representar intereses; difícilmente tienen una clientela electoral concreta, alineada por variables tales como la clase social, la región, el género, la etnia, la religión y la ecología. Complementariamente, su definición ideológica es extremadamente vaga, a la vez que dúctil, lo que lleva a una gran disociación entre la ética y el pragmatismo; la inconsistencia a la hora de mantener programas previos mínimos una vez en el poder es la nota dominante. Asimismo se percibe una enorme capacidad de establecer alianzas, cuyo carácter sería insólito según la óptica histórica nacional, pero que se suscitan tanto por el pragmatismo recién enunciado como por el alto número presente en el escenario parlamentario, cuya medida es superior a las cinco forma. ciones. A pesar de que en algunos países se siguen manteniendo identidades partidistas muy fuertes ${ }^{29}$ éstos son la minoría, y según los últimos estudios sobre el comportamiento de la opinión pública tienden a diluirse paulatinamente en algunos de los casos. En México, por el contrario, el sistema de partidos continúa manteniendo un acto hegemónico que desarrolla todavía las funciones fundamentales por las que sobrevive el sistema político. Ahora bien, aunque el papel del PRI continúa siendo muy relevante, su inconsistencia ideológica, alterada desde la cúpula dirigente, es similar a la de otros partidos en los restantes paises. Paralelamente, los demás partidos mexicanos tienen un comportamiento irregular que puede asimilarse al de otros casos latinoamericanos. El PAN mantiene un nivel constante de apoyo electoral y de coherencia programática. Por el contrario, la oferta de

29 Los casos más tradicionales en este sentido serían los de Argentina (peronistas y radicales), Colombia (conservadores y liberales), Costa Rica (liberacionistas y democristianos), Chile (democristianos, derecha y socialistas), Perú (apristas y antiapristas), Uruguay (blancos, colorados y frenteamplistas) y Venezuela (adecos y copeyanos). 
izquierda, representada por el PRD, cosechó un fracaso notable en los comicios de 1991 que hundió las expectativas levantadas tres años antes.

Junto a esta reformulación del papel partidista se registra un notable decrecimiento del poder sindical, lo que acontece por el desgaste sufrido por la militancia sindical durante los años de la represión militar, por los efectos de la crisis económica que han arrojado a la economía informal a un gran número de trabajadores cuyo encuadramiento en el entramado sindical es sumamente complicado, $y$ por el deterioro de la relación partido-sindica to que ha llevado a la ruprura de la vieja fórmula de la "correa de transmisión", circunstancia que ha generado un efecto peculiar en México, donde el nonagenario líder histórico sindical Fidel Velázquez fue reelegido una vez más en 1992, al frente del todopoderoso sindicato oficial; se trata de la inversión del papel desempeñado por el sindicato: mientras que tradicionalmente fue un recurso de transmisión de demandas de la clase trabajadora al sistema político, ahora se ha convertido en un mero organismo de control y de disciplina de dicha clase por parte del gobierno.

En cuarto término, conjuntamente con el periodo de democratización política de la pasada década, se replantea la puesta en marcha de transiciones destinadas a reemplazar la "matriz estado-céntrica" 30 vigente desde 1930 , lo que les da un carácter de extremada novedad. O si se prefiere, se trata del engarce entre las transiciones políticas

\footnotetext{
30 Cavarozzi, "Más", 1991, pp. 85-112.
}

y las económicas. Estas últimas registran una triple formulación: la imposición y el imperio incuestionable de la lógica del mercado; el achicamiento del Estado, tanto en lo que se refiere a su reforma estructural como a la privatización de amplios rubros del sector público; por último, la indiscutida y voluntaria internacionalización de la economía nacional sobre la base de la búsqueda de un espacio o de nichos en los mercados internacionales, para lo cual el comportamiento de algunos sectores de la economía nacional adquiere costas de furiosa com. petitividad, y mediante el énfasis en los redescubiertos procesos de integración subregional. Pero en aquellos países latinoamericanos en los que las transformaciones económicas de desmantelamiento del Estado nacional popular, en términos de Alain Touraine, antecedieron a las reformas políticas, lo hicieron con un intervalo de tiempo relativamente breve, circunstancia que no parece acontecer en México. En este país, el proceso de liberalización y de desregularización económica se ha realizado a un ritmo vigoroso incomparablemente mayor, tanto en velocidad como en profundiclad, que en los otros países del área. Se han tocado apartados sobre los que durante décadas se basó la iconografia popular y oficial, entre los cuales figura como más destacado la reforma profunda al artículo 27 de la Constitución, proponiéndose las adecuaciones a la configuración del sistema de tenencia de la tierra "conforme a la nueva realidad" 31 que se vive en el

31 Iniciariva de decreto del presidente Salinas 
campo mexicano. Por el contrario, las modificaciones en el ámbito democra. tizador del sistema político mexicano han sido muy reducidas $\mathrm{y}$ bastante frustrantes si se las compara con las de los otros casos.

Conjuntamente con estas cuatro situaciones nuevas que componen el escenario actual latinoamericano, aparece como elemento recurrente el retorno a la democracia electoral, cuya vigencia otrora llegó a comportar posibles soluciones a la crisis de modernización política del continente y que se mostró incapaz de establecer ligaduras lo suficientemente sólidas que llegaran a asegurar la consolidación del sistema democrático. Ahora bien, la vuelta a las pautas de la democracia electoral es directamente imposible en México, simplemente porque nunca antes existió. La necesidad de poner en marcha procesos electorales diáfanos y competitivos se alza, de esta manera, como un elemento de profunda diferenciación y marginación, junto con Cuba, frente al resto de la región, al haber traspasado ya ese umbral países como la República Dominicana y Nicaragua, quintaesencias durante décadas de estados sultanísticos.

Este escenario, que conforma a las democracias emergentes en la actualidad o, en otros términos, a los países en vías de consolidación democrática latinoamericanos, concita dos características que se complementan. La primera se refiere al déficit de. mocrático crónico previo, existente en

de Gortari ante la Cámara de Diputados el 7 de noviembre de 1991, Comercio Exterior, vol. 41, núm. 11, noviembre 1991, p. 1094. toda la región y con mucha intensidad y constancia en México, y que fue consecuencia del populismo, de los regímenes cleptocráticos centroamericanos, de la revolución como método de alcanzar el poder y del autoritarismo burocrático-autoritario. Todo este legado aportó una recurrente minusvalorización del hecho electoral que se tradujo en comicios nulamente competitivos, donde se proscribian candidatos y opciones políticas y en los que la manipulación del censo y de los resultados era la norma habitual. Como consecuencia inmediata, si lo democrático era equiparado a lo electoral y esto era sinónimo de corrupción, fraude y violencia, el descrédito de la prédica y de la praxis democrática era total, la incidencia en la cultura política de los ciudadanos y la confusión en torno a los valores democráticos fue total, hechos ambos que trajeron consigo en unos casos la inhibición y en otros el rechazo definitivo a la democracia como método. Desde la década de los años treinta y durante más de medio siglo, en México se han sucedido ininterrumpidamente elecciones cada tres años para renovar la Cámara de Diputados y cada seis años para sustituir al presidente; también se han efectuado regularmente comicios en los estados para la elección de los gobernadores y de sus asambleas legislativas. Sin embargo, su nula competitividad, la prepotencia del partido del Estado y las intervenciones caciquiles hicieron que se sedimentase y después creciese una conciencia popular que no daba crédito alguno al proceso y que provocaba la automarginación 
$y$ el recelo de la liturgia electoral, con consecuèncias que llegan hasta el momento presente.

EL PAPEL DE LOS PROCESOS ELECTORAIES Y LOS PROBLEMAS DE LAS VÍAS INSTITUCIONALISTAS

El elemento relevante $e$ incuestionable que aporta el último periodo de democratización en América Latina es el éxito en la práctica en la totalidad de los procesos electorales, donde México se alza como la gran excepción. Por primera vez, en más de cinco décadas en algunos casos (Argentina y Perú), y en general en su historia (Nicaragua, El Salvador, República Dominicana) se ha producido la alternancia política entre dos gobiernos libremente elegidos y de distinto color político, mediando el respeto a la más estricta legalidad constitucional. Además se ha agrandado notablemente el ámbito electoral, de suerte que no sólo concierne esta ampliación al marco del censo (sufragio universal completo y mayoría de edad a los 16-18 años) sino que también abarca a nuevos escenarios, insólitos hasta la fecha, como son los de las provincias y municipios, así como empresariales y sindicales. Finalmente, se constata la presencia generalizada en los diferentes sistemas políticos de una sensación de certeza en la existencia de una próxima elección en el tiempo legalmente establecido. En México, solamente este último factor parece plausible, no encontrándose en un escena. rio verosímil ni la alternancia política de la oposición ni la pureza del sufragio, que se hace casi más aguda que en el nivel nacional en los ámbitos estatales y municipales.

Lo paradójico es que México no se encuentra afectado por la cadena de éxitos técnicos de los procesos electorales, junto con periodos de estabilidad inusitada que ya se extienden por más de diez años, de otros vecinos. Por consiguiente, no parece inmerso en la tendencia, marcadamente institucionalista, ${ }^{32}$ que estŕ a punto de convertirse en creencia y que establece una relación funcional, prácticamente unívoca, y elevada a la categoría de necesaria y suficiente, entre los hábitos y mecanismos de la práctica democrática y la propia supervivencia del sistema político democrático, a pesar de la existencia de voces de alarma suficientemente sonoras y escépticas en contra de los efectos de la ingeniería política que pueden arrastrar los mecanismos electorales. ${ }^{33}$ Un hecho que avala esta situación reside en el sorprendente reforzamiento de la figura presidencial mexicana por un medio extrainstitucional, y que además apuntala aspectos hoy periclitados del Estado populista, como es el Programa Nacional de Solidaridad. Su instrumentalización exclusiva por parte del presidente lo convierte en un agente de movilización clientelar de primera magnitud que coopera en hacer menos traumáticos los efectos de las políticas neoliberales antiestatistas.

32 Diamond, Linz y Lipset, Democracry, 1989, pp. 19-30, recogen el impacto de las instituciones políticas como fuente de fracaso o de progreso del sistema democrático.

33 Nohlen, "Política", 1991, p. 170. 


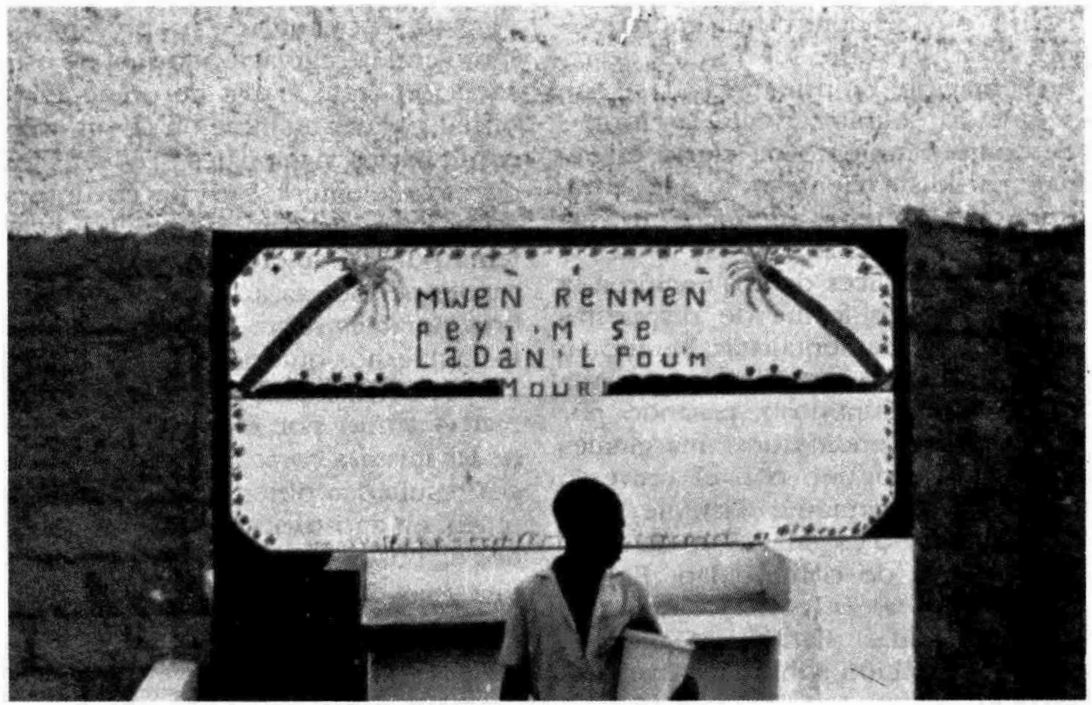

El peligro de la adopción de una visión estrictamente institucionalista es que los países latinoamericanos pueden llegar a ser extrapolados de su propia realidad socioeconómica y ascendidos a un nivel teórico de laboratorio en el que se recarguen las tintas sobre los aspectos formales del proceso político a los que se les hace casi únicos responsables de la benignidad del funcionamiento de la totalidad del sistema. Sin embargo, el caso mexicano es excepcional por cuanto que todavía no se ha traspasado este umbral inicial en el que los aspectos institucionales representan avances notables. Ahora bien, esto no puede ignorar que un estudio riguroso del ámbito latinoamericano debe tener en cuenta que no sólo hereda problemas políticos serios derivados de una determi- nada cultura política y de la forma en que se llevaron a cabo las transiciones políticas, como ya quedó indicado al inicio de la presente exposición, sino que también presenta problemas sociales y económicos similares a los del inicio de la década de 1960, con el agravamiento de haberse alcanzado un estadio de urbanización muy elevado, de pesar sobre las finanzas nacionales la carga de la deuda externa y de encontrarse la economía internacional más cerrada en tres grandes bloques.

MÉXICO TODAVÍA NO ES UN PAíS EN VÍAS DE CONSOLIDACIÓN DEMOCRÁTICA

De las tres circunstancias recién enunciadas para América Latina, el proceso de movilización social hacia las ur- 
bes es el que tiene unas implicaciones mayores sobre los niveles de representación. En primer lugar, se han sepultado definitivamente las formas de comportamiento de cierto engranaje partidista clientelar, lo que se traduce en fenómenos anómicos cuya expresión diferenciada puede ir desde unos altos índices de abstención a la integración en forma de respuestas violentas fundamentalistas, siendo su expresión más conocida la peruana de Sendero Luminoso, pasando por el apoyo a candidaturas marginales sin engarce alguno con el universo partidista previamente existente, cuyo éxito inicial no es garantía ni de asentamiento ni de continuidad. En segundo término, la localización de masas marginadas plantea un reto de dificil superación sobre la eficacia directa de políticas públicas en torno a vivienda, sanidad, transportes y educación que afectan tanto a niveles inferiores del proceso de toma de decisión política (de municipios a gobernaciones) como en el marco estatal, con el consiguiente coste negativo en el balance electoral.

En cuanto a la hipoteca de la deuda externa y a la dificultad de concretar un mejor engarce en la economía internacional, ambas condicionan las reformas emprendidas en favor del establecimiento de una economía de mercado mediante la desregularización, las privatizaciones de empresas y servicios del sector público y la utilización de políticas monetaristas indiscriminadas. En este escenario, México goza de una mejor posición de partida, toda vez que su economía se encuentra ya integrada en unos niveles muy elevados con la norteamericana, de ma- nera que ahora uno de sus problemas, junto con la regulación formal de esta situación en el marco de un acuerdo oficial, es la diversificación de sus otras contrapartes nacionales. Los efectos de estos elementos de la coyuntura económica latinoamericana en un sistema político sujeto a consultas electorales periódicas, acarrea incertidumbres notables sobre las decisiones de los gobiernos que presuponen derrotas por la ineficacia de las medidas tomadas, tanto por el erróneo diseño de las mismas como por los defectuosos resultados obtenidos. Por el contrario, en agosto-septiembre de 1991, dos elecciones parciales, las de Argentina y las de México, han sancionado la bonanza de los resultados conseguidos por las políticas gubernamentales. El comportamiento de estos resultados, en sendas situaciones, se aproxima al de otros electorados de sistemas democráticos más longevos frente a situaciones similares.

Las actuales democracias electorales latinoamericanas se engarzan a aquéllas otras que se encuentran dominadas por el principio de la incertidumbre ${ }^{34}$ en lo que se refiere a las expectativas producidas por el régimen político conformado. Todo ello es una situación típica de casos en los que se acaba de dejar o bien una prolongada situación autoritaria o bien una dilatada serie de situaciones dominadas por la inestabilidad crónica en la que formas pseudodemocráticas se alternan sucesivamente con otras autoritarias. Frente a sistemas democráti-

${ }^{34}$ O'Donnell y Schmitter, Transiciones, 1988 , pp. 15 y ss. 
cos más avanzados, o si se prefiere más consolidados, en los que la certeza es una categoría dominante, ésta es una situación que, genéricamente, está en trance de consolidación democrática, pero a la que México no ha hecho todavía sino aproximarse.

Volviendo al comienzo del presente trabajo, el reto intelectual existente es el de encuadrar, en el seno de la teoria democrática y su aplicación a los sistemas políticos, a los nuevos países que han adoptado y asentado durante más de una década, o están en trance de ha. cerlo, los procedimientos democráticos, e inferir sus características más débiles que les hacen no poder ser considerados en el ámbito reducido de los sistemas estudiados por algunos de los autores ya citados. Si se estima que los sistemas políticos para que puedan considerarse consolidados deben dar cobijo a un equilibrio entre un alto grado de institucionalización poliárquica, un nivel de eficacia suficiente para ofrecer soluciones a los problemas más generales con los que se enfrenta la población, y una aceptación por parte de la mayoría, tácita o expresa, de que las reglas del juego político establecidas son las únicas posibles hasta llegar a convertirse en una "creencia cívica", se está estableciendo un umbral para poder ubicar a los distintos sistemas nacionales en diferentes apartados.

Las denominadas democracias elec. torales pueden intégrarse en un espa. cio definido por una correcta y continuada praxis institucional poliárquica (a la que México todavía no ha llegado) que, sin embargo, se ve influenciada por el peculiar legado de la transición política llevada a cabo en los aspectos enunciados anteriormente. Pero, por el contrario, la eficacia de las diferentes políticas puestas en marcha hasta 1992 no ha conseguido los objetivos ni en asegurar el crecimiento de la riqueza nacional, ni mucho menos en disminuir la brecha existente entre la minoritaria burguesía y las masas depauperadas. Tampoco se ha conseguido ampliar el margen de confianza de la población en el sistema político, lo cual se evalúa no únicamente en la aceptación de la democracia o en la participación electoral, sino en la capacidad de fiarse del sistema, del imperio de la ley y de sus dirigentes. Todo ello define una situación peculiar, diferente de la de las democracias consolidadas donde o no aparecen o se han superado esas constricciones y que en México es aún más grave por encontrarse en un estadio previo. Esta situación es obviamente dinámica en cuanto a que las restricciones concep. tuales señaladas están sometidas a mecanismos de negociación continuos en el proceso de toma de decisiones por mor de la operatividad de la práctica poliárquica en la mayoría de las situaciones. Es por esta razón que el caso de aquellos países latinoamericanos que se incluyen dentro de estas democracias electorales puede ser definido como de países en vías de consolidación democrática, ${ }^{35}$ ámbito del que se alejan los que, como México (pero también algunos países de la Europa.Oriental), no han producido todavia una correcta operatividad electoral.

35 Alcántara Sáez, "Sobre”, 1991. 


\section{BIBLIOGRAFIA}

-Alcántara Sáez, Manuel, "¿Dónde encajan las democracias electorales latinoamericanas?" en América Latina boj', SEPLA, Facultad de Ciencias Políticas y Sociología, Madrid, núm. 2, noviembre 1991, pp. 9-13.

_-, "Sobre el concepto de países en vías de consolidación democrática en América Latina", Revista de Estudios Políticos, Madrid, núm. 74, octubre-diciembre 1991.

Cavarozzi, Marcelo, "Más allá de las transiciones en América Latina", Revista de Estudios Políticos, Madrid, núm. 74, octubre-diciembre 1991, pp. 85-112.

-Collier, Ruth Berins y David Collier, Sbaping the political arena, Princeton University Press, New Jersey, 1991.

-Dahl, Robert A., La poliarquta. Partictpación y oposición, Tecnos, Madrid, 1989.

-Diamond, Larry, Juan J. Linz y Seymor Martin Lipset, Democracy in developing countries. Latin Amertca, Lynne Rienner Publishers, Boulder, 1989.

-Duverger, Maurice, Instituciones politi. cas $y$ derecho constitucional, Editorial Ariel, Barcelona, 1970.

-Gil, Federico G., Instituciones y desa. mollo político de Amértca Latina, INTAL, Buenos Aires, 1966.

-Hartlyn, Jonathan, The politics of coalition rule in Colombia, Cambridge University Press, Cambridge, 1988.

-Huntington, Samuel P., Political order in changing societies, Yale University Press, New Haven, 1968.

-Lambert, Jacques, América Latina. Es. tructuras sociales e instituciones politicas, Editorial Ariel, Barcelona, 1973.
-Lanning, Eldon, "A typology of latin american political systems", Comparative Politics, vol. vI, núm. 3, abril 1974.

-Lijphart, Arend, Las democracias contemporáneas, Editorial Ariel, Barcelona, 1987.

-Lipset, Seymour Martin, El bombre político, Editorial Universitaria, Buenos Aires, 1977.

-McDonald, Ronadl H., y J. Mark Ruhl, Party politics and elections in Latin Ame. rica, Westview Press, Boulder, 1989.

-Needler, Martin C., Political sjistems of Latin America, D. Van Nostrand Co. Ptinceton, 1964.

-Nohlen, Dieter, "La política de la reforma electoral", La reforma electoral en Latinoamérica. Memorias del IV Curso anual interamericano de elecciones, IIDHCAPEL, San José de Costa Rica, 1991.

-O'Donnell, Guillermo y Phillipe C. Schmitter, Transiciones desde un gobiemo autoritatio. Conclusiones sobre las demo. cracias inciertas, Editorial Paidós, Buenos Aires, 1988.

-Prélot, Marcel y Jean Boulouis, Institutions politiques et drolt constitutionnel, Dalloz, París, 1987.

-Skidmore, Thomas E. y Peter H. Smith, Modern Latinoamerica, Oxford University Press, Oxford, 1989.

-Touraine, Alain, América Latina. Política y sociedad, Espasa Calpe, Madrid, 1989.

-Varela, Gonzalo, "El análisis de los sistemas políticos latinoamericanos", Secuencia, núm. 16, enero-abril 1990, pp. 137. 148.

-Wiarda, Howard J. y Harvey F. Kline, Latin american politics and development, Westview Press, Boulder, 1990. 